\title{
A Novel Neobladder-Urethral Drag-and-Bond Anastomosis Technique During Laparoscopic Radical Cystectomy for Ileal Orthotopic Neobladder: Surgical Technique and Initial Research
}

\author{
Zhaojun $\mathrm{Yu}^{\mathrm{I}-3}$ \\ Jianbiao Huang ${ }^{1-3}$

'Department of Urology, The Second Affiliated Hospital of Nanchang University, Nanchang, 330006, People's Republic of China; ${ }^{2}$ Department of Urology, The People's Hospital Affiliated to Nanchang University, Nanchang, 330006, People's Republic of China; ${ }^{3}$ Medical Department, Nanchang University, Nanchang, 330006, People's Republic of China; ${ }^{4}$ Department of Ultrasound, The First Affiliated Hospital of Nanchang University, Nanchang, 330006, People's Republic of China
Correspondence: Tao Zeng

Department of Urology, The Second Affiliated Hospital of Nanchang

University, No. I Minde Road, Nanchang, Jiangxi Province, 330006, People's

Republic of China

$\mathrm{Tel}+861360791917 \mid$

Email Taozeng40709@sina.com
This article was published in the following Dove Press journal: Cancer Management and Research

Purpose: To explore the application of the neobladder-urethral drag-and-bond anastomosis technique in laparoscopic radical cystectomy (LRC) with ileal orthotopic neobladder (IONB) reconstruction.

Patients and Methods: This is a retrospective cohort study on a procedure performed by a single surgeon. From January 2014 to December 2018, we identified 43 male bladder cancer patients who received LRC with IONB reconstruction. These patients were divided into two groups, with 22 patients undergoing neobladder-urethral drag-and-bond anastomosis (NUDA) and 21 patients undergoing neobladder-urethral anastomosis under laparoscopy (NUAL). Anastomosis time, catheter removal time, postvoid residual (PVR), maximum urinary flow rate (Q-max), urine leakage and anastomotic stenosis were used to evaluate the simplicity and surgical effect of the two groups.

Results: Both groups demonstrated similar tumor characteristics. A significant difference in neobladder-urethral anastomosis time was found between the NUDA group and the NUAL group ( $14.6 \pm 0.4$ vs $70 \pm 2.5 \mathrm{~min}, \mathrm{P}<0.0001)$, and there was no significant difference in other characteristics.

Conclusion: The neobladder-urethral drag-and-bond anastomosis technique in LRC and IONB reconstruction, with its shorter learning curve, was easier and more convenient than neobladder-urethral anastomosis under laparoscopy.

Keywords: drag-and-bond anastomosis, neobladder-urethral anastomosis, laparoscopic radical cystectomy, ileal orthotopic neobladder, bladder cancer

\section{Introduction}

Radical cystectomy (RC) is an effective method for the treatment of invasive bladder cancer while ileal orthotopic neobladder (IONB) reconstruction is an ideal method of urinary diversion. ${ }^{1,2}$ Due to the narrow and deep operation space behind retropubic, it is difficult to hold, insert, and withdraw the needle ${ }^{3}$ If the neobladder-urethral anastomosis has some tension, the anastomosis will be more tedious. Therefore, neobladder-urethral anastomosis is considered as an important and critical step in these procedures. We designed a novel technique to "anastomose" the urethra with the neobladder by using only one 20 Fr Foley catheter, 
which was simple but effective. This study presented our initial research on this new anastomosis between neobladder and urethra in laparoscopic radical cystectomy (LRC) with ileal orthotopic neobladder (IONB) reconstruction.

\section{Patients and Methods}

\section{Patients}

We retrospectively analyzed the clinical data of 43 patients with bladder cancer who underwent LRC with IONB in the People's Hospital Affiliated to Nanchang University from January 2014 to December 2018. Among them, 22 patients received neobladder-urethral drag-and-bond anastomosis (NUDA) and 21 patients received the neobladder-urethral anastomosis under laparoscopy (NUAL). In order to determine the diagnosis of invasive bladder cancer, all patients underwent detailed preoperative examination including biochemical examination, electrocardiogram, X-ray, urinary system color ultrasound, pelvic CT and cystoscope biopsy. The inclusion criteria were as follows: 1) patients with LRC and performed by the same surgical team from January 2014 to December 2018; 2) the neobladderurethral anastomosis was the neobladder-urethral anastomosis under laparoscopy or drag-and-bond anastomosis; 3) patients with 3 months follow-up: The exclusion criteria were as follows: 1) LRC and IONB performed by a different surgical team; 2) other neobladder-urethral anastomosis styles; 3) LRC and IONB for non-bladder cancer; 4) the follow-up was less than 3 months. All patients were performed the surgery by the same surgical team to avoid bias due to differences in surgical skills and experience.

\section{Surgical Technique}

Laparoscopic Radical Cystectomy (LRC)

Patients were in supine position, with head raised $30^{\circ}$ and intubated under general anesthesia. We established five surgical operation channels and kept the urethral length of the apex of prostate $1 \mathrm{~cm}$ and performed standard pelvic lymph node dissection (PLND) in LRC.

\section{Ileum Orthotopic Neobladder (IONB) Reconstruction}

We made an $8 \mathrm{~cm}$ midline incision under the umbilicus and removed the specimen after finishing LRC. A $45-\mathrm{cm}$ distal ileal segment was isolated, folded into a "W" configuration and constructed a spherical neobladder. Then, the continuity of the ileum was restored. Bilateral ureters were then anastomosed to the base of the neobladder in an anti-reflux manner. The 6-Fr double-J stents were left on each side.

\section{Anastomosis of the Neobladder and Urethra \\ The Drag-and-Bond Anastomosis Technique}

Our neobladder-urethral drag-and-bond anastomosis technique was used in the NUDA group. In NUDA group, after we completed the LRC and IONB, a hole similar in diameter to that of a 20 Fr Foley catheter was created at the apex of the neobladder, and the anterior wall of the neobladder was sutured. The 20 Fr Foley catheter was inserted into the pelvis through the urethra (Figure 1A). Six to eight windings of 2-0 Mersilk were wrapped around the Foley catheter $0.5 \mathrm{~cm}$ distal to the catheter balloon and tied. The catheter was inserted into the neobladder through the hole at the apex. The hole with the catheter windings was sutured with 2-0 VICRYL at $6,8,10,12,2$ and 4 o'clock intermittently (Figures 1B and 2). The neobladder was secured to the Mersilk wound around the catheter with a 2-0 VICRYL suture (Figure 1C). The catheter balloon was inflated with $20 \mathrm{~mL}$ normal saline, and gentle traction was applied to the Foley catheter. The neobladder descended to the lowest position of the pelvic cavity along the direction of the catheter and naturally approached the urethra. The catheter was gently pulled outward with proper tension. Plain gauze was tied around the catheter and slid to the outer urethral orifice (Figure 1D) (Supplementary Video 1 and Supplementary Video 2).

In NUAL group, after the LRC and IONB were completed, the pneumoperitoneum was re-established, and neobladder-urethral anastomosis was carried out under laparoscopy. The outcomes of these patients were analyzed retrospectively.

\section{Follow-Up}

After the treatment, we carried out postoperative review on the patients every month, the review items included urinary system color doppler ultrasound, cystoscopy and urethrography. The postoperative follow-up period was 3 months.

\section{Parameters and Endpoint}

The observation indicators of this study were as follows. First, the baseline parameters of the patients included gender, age, tumor T stage, pathological type, pathology grade, maximum tumor diameter, tumor number; Second, intraoperative observation indicators included: time for urinary diversion excluding neobladder-urethral anastomosis, 

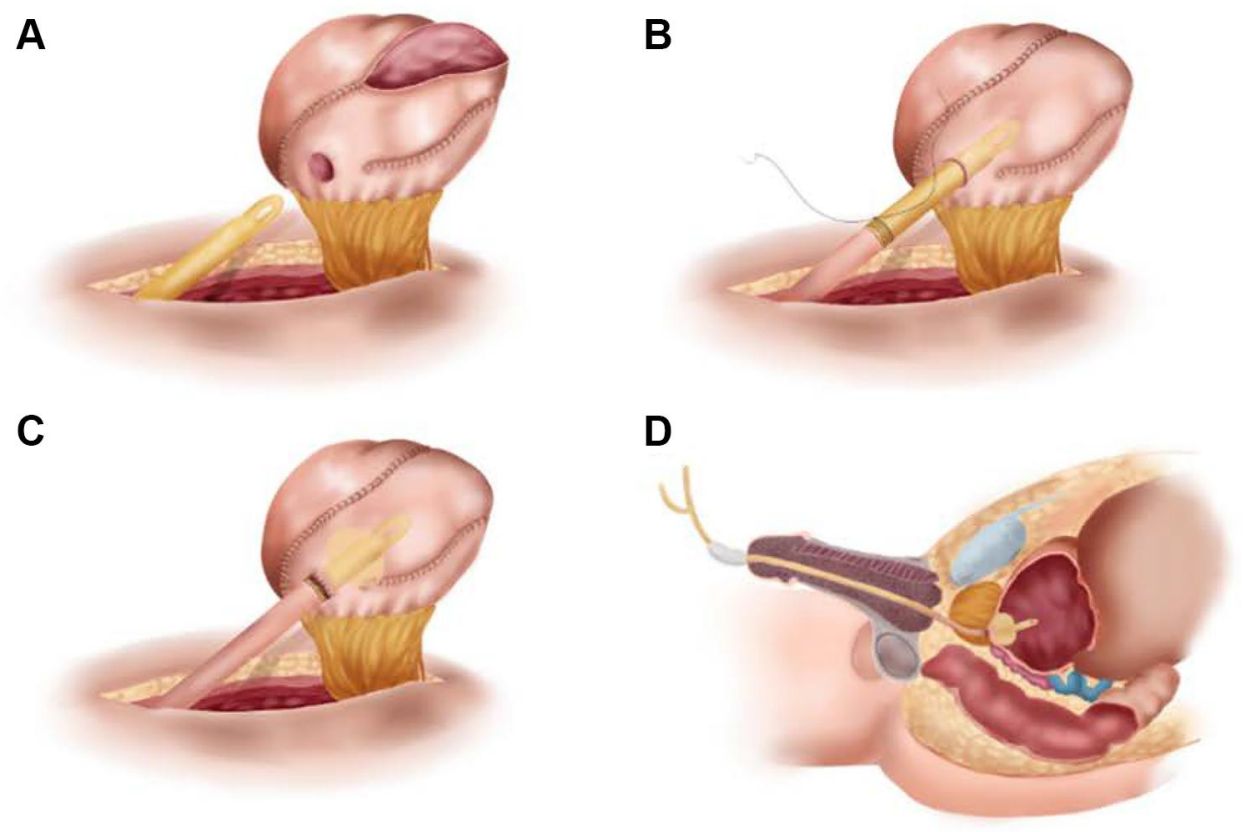

Figure I (A) A hole similar in diameter to that of a 20 Fr Foley catheter was created at the apex of the neobladder, and the anterior wall of the neobladder was sutured. The $20 \mathrm{Fr}$ Foley catheter was inserted into the pelvis through the urethra. (B) Six to eight windings of 2-0 Mersilk were wrapped around the Foley catheter $0.5 \mathrm{~cm}$ distal to the catheter balloon and tied. The catheter was inserted into the neobladder through the hole at the apex. The hole with the catheter windings was sutured with $2-0$ VICRYL at 6, 8, I0, 12, 2 and 4 o'clock intermittently. (C) The neobladder was secured to the Mersilk wound around the catheter with a 2-0 VICRYL suture. (D) The catheter balloon was inflated with $20 \mathrm{~mL}$ normal saline, and gentle traction was applied to the Foley catheter. The neobladder descended to the lowest position of the pelvic cavity along the direction of the catheter and naturally approached the urethra. The catheter was gently pulled outward with proper tension. Plain gauze was tied around the catheter and slid to the outer urethral orifice.

neobladder-urethral anastomosis time; Third, postoperative observation indicators included catheter removal time, PVR and Q-max after 3 months of surgery, the incidence of urine leakage and anastomotic stenosis after 3 months of surgery. We estimated PVR through the B-ultrasound, assess urine leakage by urethrography and evaluate the anastomosis stenosis by cystoscopy.

\section{Statistical Analysis}

GraphPad Prism 7.00 software was used for statistical analysis of all data. All quantitative data were represented by $\mathrm{X} \pm \mathrm{S}$, and comparison between groups was performed by unpaired $T$ test or Mann-Whitney test. The count data were represented by example (n) and frequency (\%), and the Chi-square test or Fisher's exact test was used for intergroup comparisons, $\mathrm{P}<0.05$ was considered to be statistically significant.

\section{Results}

Our research retrospectively analyzed the clinical data of 43 invasive bladder cancer patients. Among them, 21 patients were treated with the neobladder-urethral anastomosis laparoscopically, the other 22 patients were treated with the neobladder-urethral drag-and-bond anastomosis. All patients were male. Tumor characteristics of the two groups are shown in Table 1. There were no statistically differences in tumor $\mathrm{T}$ stage, pathology grade, tumor number, maximum tumor diameter and pathological type between the two groups (all $\mathrm{P}>0.05$ ).

All patients in the two groups were not transferred to open surgery. The intraoperative and postoperative characteristics of the two groups are shown in Table 2. The neobladder-urethral anastomosis time was significantly shorter in NUDA group than that in NUAL group (14.6 \pm 0.4 vs $70 \pm 2.5 \mathrm{~min}, \mathrm{P}<0.0001$ ), but there was no statistical difference between the two groups in terms of time for urinary diversion excluding neobladder-urethral anastomosis $(126.5 \pm 2.1$ vs $125.4 \pm 2.1 \mathrm{~min}, \mathrm{P}=0.718)$. Foley catheter removal time, PVR and Q-max between the two groups showed no significant difference (19.45 \pm 0.2347 vs $19.33 \pm 0.261$ days, $\mathrm{P}=0.795,21.4 \pm 6.2$ vs $23.3 \pm 7.9 \mathrm{~mL}, \mathrm{P}=0.667,19.6 \pm 0.7$ vs $18.9 \pm 0.8 \mathrm{~mL} / \mathrm{s}$, $\mathrm{P}=0.362$ ). In terms of urine leakage after surgery, 2 patients in NUDA group and 3 patients in NUAL group had urine leakage, showing no statistical difference $(\mathrm{P}=0.595)$. Anastomotic stenosis occurred in 1 patient in 
A

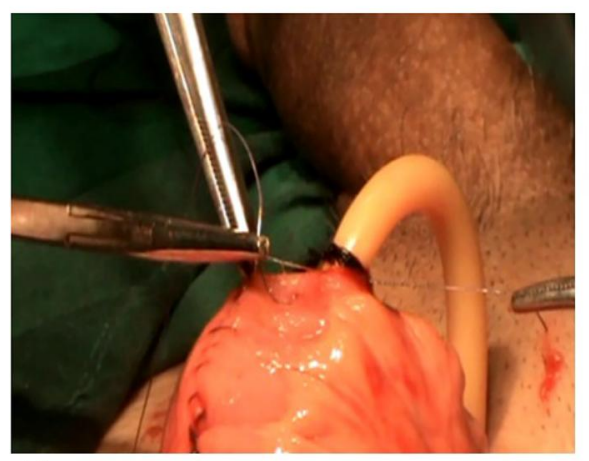

D

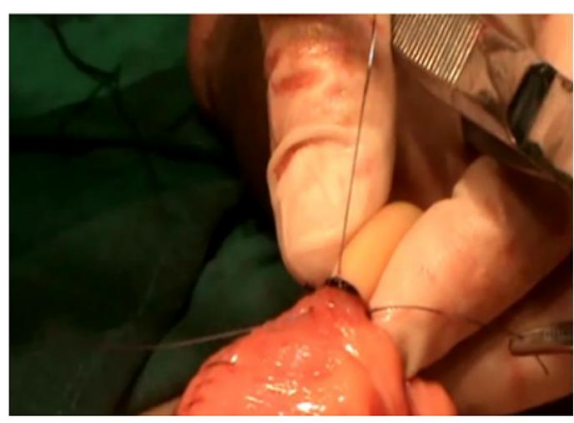

B

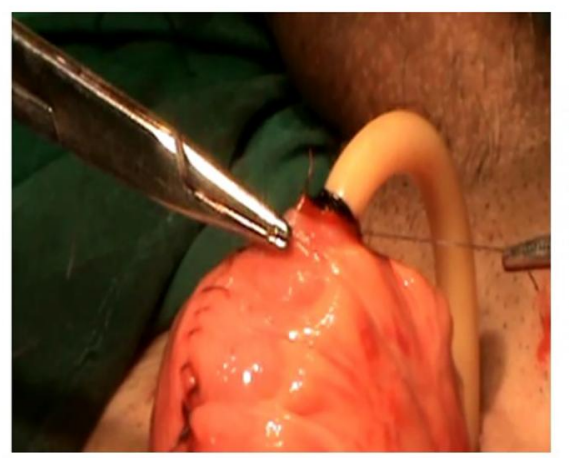

E

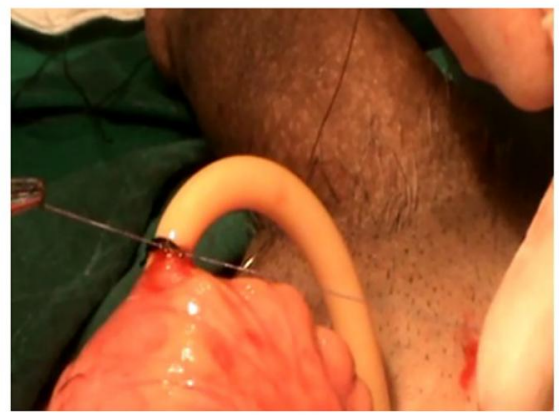

C

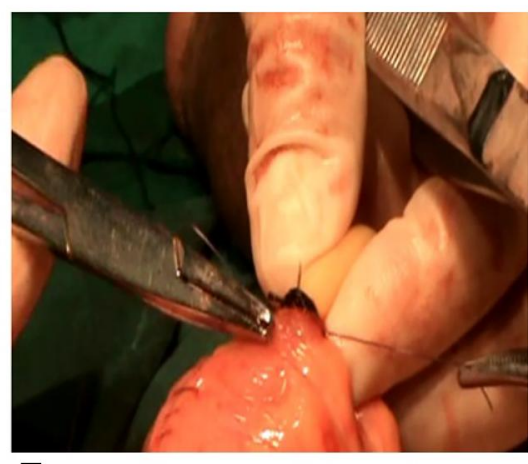

$\mathbf{F}$

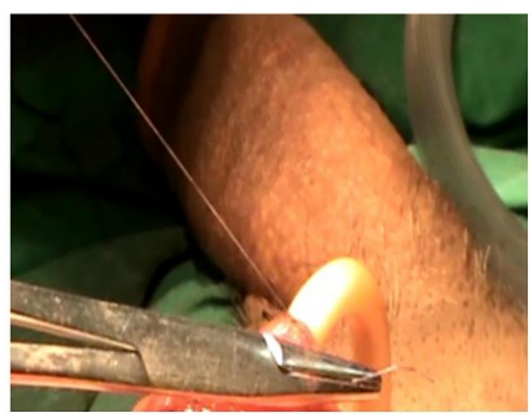

Figure 2 Operation chart: The hole of the neobladder was sutured intermittently with the catheter windings. (A and B) The needle piercing through the wall of the neobladder and coming out of the hole; (C) The needle passing through the catheter windings; (D-F) The suture being tied in a surgical knot.

each group, respectively ( $\mathrm{P}>0.9999)$. The anastomotic area healing under cystoscope is shown in Figure 3. The follow-up period was 3 months.

\section{Discussion}

Neobladder-urethral anastomosis is technically challenging whatever its laparoscopic surgery, robot-assisted surgery or open surgery in radical cystectomy and orthotopic neobladder. ${ }^{4}$ Due to the narrow and deep operation space behind retropubic, there is insufficient visualization of the anastomotic site and the instability of the neobladderurethra itself compared with the native bladder. ${ }^{3}$ If the overweight and obese patients have a tensional mesentery, the neobladder-urethral anastomosis is technically harder. ${ }^{5}$ It is very difficult to master the key step and the learning curve is very long for most surgeons. Herein, we reported a novel procedure, a neobladder-urethral drag-and-bond "anastomosis" technique to facilitate neobladder-urethral anastomosis.

Many surgeons used different methods to perform anastomosis of the neobladder and urethra after completing the procedures of LRC and IONB reconstruction. There were some surgeons who made a midline incision in the lower abdomen, removed the specimen and constructed the neobladder extracorporeally. Then, they re-established the pneumoperitoneum and performed the suture of anastomosis between neobladder and urethra under laparoscopy. ${ }^{6}$ Re-establishing the pneumoperitoneum is complicated and the procedure for the anastomosis between neobladder and urethra is difficult, timeconsuming and tedious under laparoscopy. Some surgeons who performed RC and extended PLND under laparoscopy performed orthotopic neobladder reconstruction and the neobladder-urethral anastomosis through open surgery. ${ }^{7}$ Open surgery is not only traumatic, but also difficult to perform because of no easy exposing the field of vision.

Menon et al carried out LRC and PLND by the DaVinci Surgical System ${ }^{8}$ and the specimen was entrapped in a bag and removed through a 5-6 cm suprapubic incision. A different surgical team exteriorized the bowel through this incision and created a neobladder extracorporeally. The neobladder was internalized, the incision closed, re-established the pneumoperitoneum and the primary surgeon completed the neobladder-urethral anastomosis with robotic assistance. The anastomosis between the neobladder and the urethra was accurate and convenient because the DaVinci robotic surgical system has 
Table I Tumor Characteristics

\begin{tabular}{|c|c|c|c|}
\hline Characteristics & $\begin{array}{l}\text { NUDA Group } \\
(n=22)\end{array}$ & $\begin{array}{l}\text { NUAL Group } \\
(n=21)\end{array}$ & $\mathbf{P}$ \\
\hline Age (years) & $59.4 \pm 1.9(43-72)$ & $57.7 \pm 2.2(38-7 \mid)$ & 0.546 \\
\hline \multicolumn{4}{|l|}{ Tumor Stage } \\
\hline T0, Ta, Tis, TI & 0 & 0 & \multirow[t]{6}{*}{0.525} \\
\hline $\mathrm{T} 2 \mathrm{a}$ & $2(9.1 \%)$ & $3(14.3 \%)$ & \\
\hline $\mathrm{T} 2 \mathrm{~b}$ & $6(27.3 \%)$ & 7 (33.3\%) & \\
\hline T3a & $2(9.1 \%)$ & $4(19.1 \%)$ & \\
\hline T3b & $12(54.5 \%)$ & 7 (33.3\%) & \\
\hline $\mathrm{T} 4$ & 0 & 0 & \\
\hline \multicolumn{4}{|l|}{ Pathological type } \\
\hline $\begin{array}{l}\text { Transitional cell } \\
\text { carcinoma }\end{array}$ & 19 (86.4\%) & 18 (85.7\%) & \multirow[t]{3}{*}{0.998} \\
\hline Glandular carcinoma & $2(9.1 \%)$ & $2(9.5 \%)$ & \\
\hline Squamous carcinoma & I (4.5\%) & I (4.8\%) & \\
\hline \multicolumn{4}{|l|}{ Pathology grade } \\
\hline GI & $3(13.6 \%)$ & I (4.8\%) & \multirow[t]{3}{*}{0.536} \\
\hline G2 & $8(36.4 \%)$ & $10(47.6 \%)$ & \\
\hline G3 & II (50.0\%) & $10(47.6 \%)$ & \\
\hline $\begin{array}{l}\text { Maximum tumor } \\
\text { diameter }(\mathrm{cm})\end{array}$ & $3.0 \pm 0.2$ & $2.9 \pm 0.2$ & 0.690 \\
\hline Tumor number & $2.5 \pm 0.4$ & $2.2 \pm 0.3$ & 0.704 \\
\hline
\end{tabular}

a magnified $3 \mathrm{D}$ vision with a surgeon-controlled camera, mechanical wrists with seven degrees of motion, motion scaling and tremor reduction. ${ }^{9}$ But the pneumoperitoneum was re-established, the machine operating arm was installed again. These steps are time-consuming and cumbersome. ${ }^{10,11}$ More importantly, the DaVinci surgical operating system was relatively expensive for the lowervolume hospitals.

Shawn Dason showed that robot-assisted radical cystectomy (RARC) with intracorporeal urinary diversion was a feasible option for patients with bladder cancer. ${ }^{12}$ Although total intracorporeal urinary diversion in RARC has been increasingly advocated in a few high-volume centers, the diversion is very complicated and requires a high level of skill on the part of the surgeon. ${ }^{13,14}$ In total, intracorporeal ileal neobladder reconstruction technique, which has long operation time and long learning curve, increases the complexity of the procedure. ${ }^{15,16}$

We performed LRC and PLND, made an incision, removed the specimen and used our novel procedure that the neobladder and the urethral stump were closed without suture after we completed LRC and the reconstruction of IONB extracorporeally. This procedure can accurately control the needle distance and margin under direct vision and decrease the risk of intraoperative and postoperative complications such as urine leakage and anastomotic stenosis. It has the following advantages: First, this novel procedure was safer and effective with the shorter time of neobladder-urethral anastomosis. It only took an average of $14.6 \mathrm{~min}$ for the whole anastomosis procedure. Second, the neobladder-urethral anastomosis was extracorporeally performed under direct vision. We did not need to reestablish a pneumoperitoneum. So, this new method, with short learning curve, is simpler and timesaving compared to the neobladder-urethral anastomosis under

Table 2 Operative and Postoperative Characteristics

\begin{tabular}{|c|c|c|c|}
\hline Characteristics & NUDA Group $(n=22)$ & NUAL Group $(n=2 I)$ & $\mathbf{P}$ \\
\hline Anastomosis time (min) & $14.6 \pm 0.4$ & $70 \pm 2.5$ & $<0.0001$ \\
\hline \multicolumn{4}{|l|}{ Time for urinary diversion } \\
\hline Excluding neobladder-urethral & $126.5 \pm 2.1$ & $125.4 \pm 2.1$ & 0.718 \\
\hline \multicolumn{4}{|l|}{ Anastomosis (min) } \\
\hline Catheter removal time (day) & $19.5 \pm 0.2$ & $19.3 \pm 0.3$ & 0.795 \\
\hline PVR (mL) & $21.4 \pm 6.2$ & $23.3 \pm 7.9$ & 0.667 \\
\hline Q-max $(\mathrm{mL} / \mathrm{s})$ & $19.6 \pm 0.7$ & $18.9 \pm 0.8$ & 0.362 \\
\hline Urine leakage & $2(0.1 \%)$ & $3(0.1 \%)$ & 0.595 \\
\hline Anastomotic stenosis & I (0.05\%) & I (0.05\%) & $>0.9999$ \\
\hline
\end{tabular}



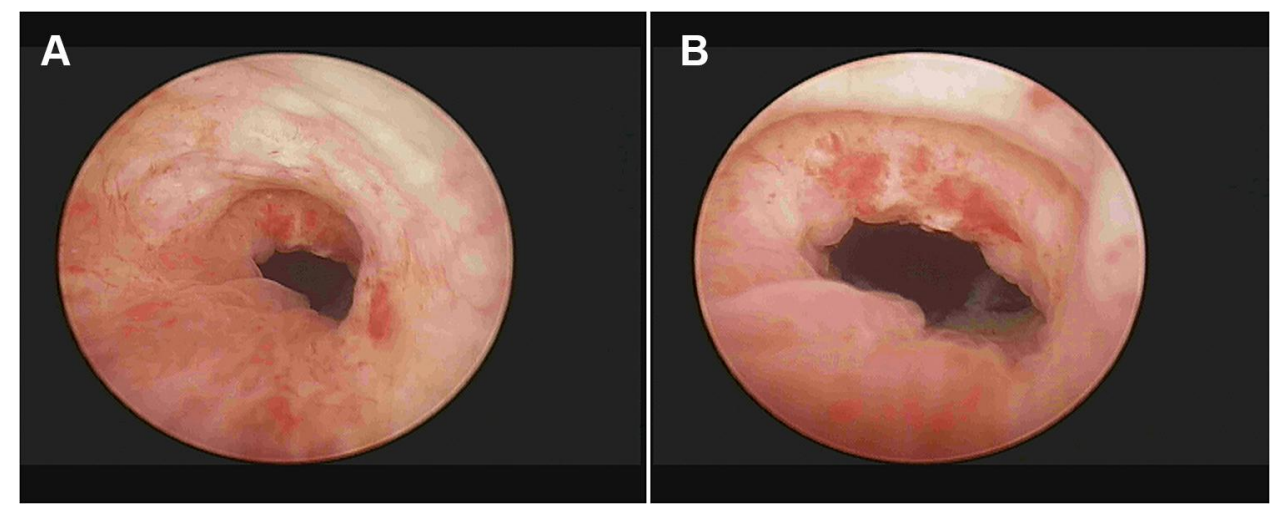

Figure 3 The anastomotic area of the patient 3 months after surgery under cystoscopy. The two images (A, B) show that there was no anastomotic stricture or neoplasm in the anastomotic area, and the mucosa of the anastomotic area was smooth and flat.

laparoscopy. It is suitable not only for LRC, but also for RARC as well as open surgery. However, some obese patients with tensional mesenteric and male patients with deep pelvis may be selected with high priority to this procedure. Finally, the new anastomosis technique would reduce the operating time but the risk of the associated complications was no increase.

Our studies also had some shortcomings. First, our study was a small sample and single-center study. Secondly, the observation characteristics of this study were not comprehensive enough, patients' data were incomplete, such as body mass index, sexual function, gastrointestinal recovery time; In the future, we will design a multi-center, large sample size, comprehensive prospective and randomized control research to achieve more reliable results.

\section{Conclusion}

This novel procedure, which we named as neobladderurethral drag-and-bond anastomosis technique, simplifies the surgical steps and considerably shortens the operating time for neobladder-urethral anastomosis. Therefore, we believe that the novel neobladder-urethral anastomosis, with its shorter learning curve, is easier and more convenient than neobladder-urethral anastomosis under laparoscopy.

\section{Abbreviations}

IONB, ileal orthotopic neobladder; LRC, laparoscopic radical cystectomy; NUDA, neobladder urethral drag-andbond anastomosis; NUAL, neobladder-urethral anastomosis under laparoscopy; PVR, post-void residual; PLND, pelvic lymph node dissection; Q-max, maximum urinary flow rate; RC, radical cystectomy; RARC, robot-assisted radical cystectomy.

\section{Ethics Statement}

Ethical approval for this study was obtained from the Ethics Committee of The People's Hospital Affiliated to Nanchang University. Since several patients had low literacy level and did not understand enough about disease, they authorized their direct relatives as legal representatives. Informed consent was obtained from all patients or their appointed representatives in accordance with the ethics committee guidelines. This study was conducted in accordance with the Declaration of Helsinki.

\section{Acknowledgments}

We are very grateful to patients who agreed to provide clinical data to us.

\section{Author Contributions}

TZ had full access to all the data in the study and took responsibility for the integrity of the data and the accuracy of the data analysis. TZ, ZJ-Y contributed to the study concept and design. TZ, ZJ-Y, ZH-Z, JB-H, HH-D, HC-C, LH-D contributed to the acquisition of data, analysis, and interpretation of data. TZ, ZJ-Y and LH-D did the drafting of the manuscript and critical revision of the manuscript for important intellectual content. ZJ-Y did the statistical analysis. All authors made a significant contribution to the conception, study design, surgery, acquisition of data, analysis and interpretation, or in all these areas; took part in drafting, revising, critically reviewing the article; gave final approval of the version to be published; have agreed on the journal to which the article has been submitted; and agree to be accountable for all aspects of the work. 


\section{Funding}

This research was supported by the National Nature Science Foundation of China (grants: 81860455) and the Jiangxi Provincial Department of Science and Technology (grants: 20161BBG70130, 20192BBGL70029).

\section{Disclosure}

The authors report no conflicts of interest in this work.

\section{References}

1. Castillo OA, Vitagliano G, Vidal-Mora I. [Laparoscopic radical cystectomy. The new gold standard for bladder carcinoma?]. Arch Esp Urol. 2009;62(9):737-744. doi:10.4321/s0004-06142009000900009.Spanish.

2. Zhong H, Shen Y, Yao Z, et al. Long-term outcome of spiral ileal neobladder with orthotopic ureteral reimplantation. Int Urol Nephrol. 2020;52(1):41-49. doi:10.1007/s11255-019-02296-x

3. Pham KN, Sack BS, O'Connor RC, et al. V-Loc urethro-intestinal anastomosis during robotic cystectomy with orthotopic urinary diversion. Can Urol Assoc J. 2013;7(11-12):E663-E666. doi:10.5489/cuaj.508

4. Miki J, Yanagisawa T, Tsuzuki S, Kimura T, Kishimoto K, Egawa S. Improved technique for intracorporeal neobladder-urethral anastomosis in laparoscopic radical cystectomy. Int J Urol. 2017;24(4):330-331. doi:10.1111/iju.13299

5. Badawy AA, Saleem MD, El-Baset EA, Morsi ES. Decreasing operative time and incontinence rates in patients treated with radical cystectomy and urethral diversion: a prospective randomized trial using a new suturing device (CAPIO). Int Urol Nephrol. 2012;44(3):769-774. doi:10.1007/s11255-011-0105-9

6. Pruthi RS, Wallen EM. Robotic-assisted laparoscopic radical cystoprostatectomy. Eur Urol. 2008;53(2):310-322. doi:10.1016/j. eururo.2007.03.067

7. Haber GP, Campbell SC, Colombo JR Jr., et al. Perioperative outcomes with laparoscopic radical cystectomy: "pure laparoscopic" and "open-assisted laparoscopic" approaches. Urology. 2007;70 (5):910-915. doi:10.1016/j.urology.2007.07.012
8. Menon M, Hemal AK, Tewari A, et al. Nerve-sparing robot-assisted radical cystoprostatectomy and urinary diversion. BJU Int. 2003;92 (3):232-236. doi:10.1046/j.1464-410X.2003.04329.x

9. Challacombe BJ, Bochner BH, Dasgupta P, et al. The role of laparoscopic and robotic cystectomy in the management of muscle-invasive bladder cancer with special emphasis on cancer control and complications. Eur Urol. 2011;60(4):767-775. doi:10.1016/j. eururo.2011.05.012

10. Albisinni S, Veccia A, Aoun F, et al. A systematic review and meta-analysis comparing the outcomes of open and robotic assisted radical cystectomy. Ital J Urol Nephrol. 2019;71(6):553-568. doi:10.23736/S0393-2249.19.03546-X

11. Castillo O, Vidal-Mora I, Rodriguez-Carlin A, Silva A, Schatloff O, Borgna V. Modified urethrovesical anastomosis during robot-assisted simple prostatectomy: technique and results. Prostate Int. 2016;4 (2):61-64. doi:10.1016/j.prnil.2016.04.001

12. Dason S, Goh AC. Contemporary techniques and outcomes of robotic cystectomy and intracorporeal urinary diversions. Curr Opin Urol. 2018;28(2):115-122. doi:10.1097/MOU.0000000000000472

13. Jonsson MN, Adding LC, Hosseini A, et al. Robot-assisted radical cystectomy with intracorporeal urinary diversion in patients with transitional cell carcinoma of the bladder. Eur Urol. 2011;60 (5):1066-1073. doi:10.1016/j.eururo.2011.07.035

14. Smith AB, Raynor M, Amling CL, et al. Multi-institutional analysis of robotic radical cystectomy for bladder cancer: perioperative outcomes and complications in 227 patients. J Laparoendosc Adv Surg Tech A. 2012;22(1):17-21. doi:10.1089/lap.2011.0326

15. Almassi N, Zargar H, Ganesan V, Fergany A, Haber GP. Management of challenging urethro-ileal anastomosis during robotic assisted radical cystectomy with intracorporeal neobladder formation. Eur Urol. 2016;69(4):704-709. doi:10.1016/j.eururo.2015.09.037

16. Koie T, Ohyama C, Makiyama K, et al. Utility of robot-assisted radical cystectomy with intracorporeal urinary diversion for muscle-invasive bladder cancer. Int J Urol. 2019;26(3):334-340. doi:10.1111/iju. 13900

\section{Publish your work in this journal}

Cancer Management and Research is an international, peer-reviewed open access journal focusing on cancer research and the optimal use of preventative and integrated treatment interventions to achieve improved outcomes, enhanced survival and quality of life for the cancer patient.
The manuscript management system is completely online and includes a very quick and fair peer-review system, which is all easy to use. Visit http://www.dovepress.com/testimonials.php to read real quotes from published authors. 